\title{
Unsupervised and online place recognition for mobile robot based on local features description
}

\begin{abstract}
Place recognition approaches have been used for solving topological mapping and localization problems. These approaches are usually performed in supervised and offline mode. In this paper, a robust appearance-based unsupervised and online place recognition algorithm, which is inspired from online sequential clustering methods, is introduced. This method combines several image features using Speedup Robust Features (SURF) by accumulating them into a union form of features inside each place cluster. In this method, the mobile robot captures omnidirectional visual information and converts them into topological place clusters. Experimental results show the robustness, accuracy, and efficiency of the method as well as its ability to create topological place clusters for solving mapping and qualitative localization problems. The performance of the developed system is remarkable in term of recognition precision performance.
\end{abstract}

Keyword: Place recognition; SURF features; Online clustering; Environment modeling; Topological localization 